\title{
Role of PhoP-PhoQ Two-Component System in Biofilm Formation of the Phytopathogen Dickeya dadantii Strain 3937
}

\author{
Md. Manjurul Haque* and Shinji Tsuyumu \\ Dept. of Science of Biological Environment, Lab. of Plant Pathology, Fac. of Agriculture, Shizuoka University, \\ 836 Ohya, Suruga-ku, Shizuoka 422-8529, Japan \\ *Corresponding author: haque59@hotmail.com
}

\begin{abstract}
To elucidate the role of PhoP-PhoQ two-component system in biofilm formation by the phytopathogen Dickeya dadantii (formerly Erwinia chrysanthemi) strain 3937, we used marker-exchanged mutants deficient in both phoP and $p h o Q$. A biofilm-inducing medium, salt-optimized broth plus glycerol (SOBG) which contains salt-optimized broth (SOB) plus $2 \%$ of glycerol, supported biofilm formation by $D$. dadantii strain 3937 to a greater extent than either M63 glycerol minimal medium or yeast extract peptone (YP) medium or Luria-Bertani (LB) medium. In addition, magnesium greatly induced biofilm formation. It was found that both the $p h o P$ and $p h o Q$ mutants exhibited enhanced ability to form a biofilm on the surface of the glass test tube as compared to the wild-type strain in SOBG medium containing high concentration of magnesium. In addition, under high magnesium condition, both the mutants produced elevated level of exopolysaccharide. Accordingly, genes responsible for exopolysaccharide production ( $w e a P$, wza and $w z c$ ) were derepressed in the mutants. These results suggest that the PhoP-PhoQ two-component system may regulate formation of biofilm, at least in part, by transcriptional control of genes responsible for exopolysaccharide biosynthesis of $D$. dadantii strain 3937. Since biofilm-associated bacteria showed more tolerance to acidic $\mathrm{pH}$ and high osmotic stress, it is apparent that the formation of biofilm may be an important factor for the survival of $D$. dadantii strain 3937 in unfavorable environment.
\end{abstract}

Keywords: PhoP-PhoQ two-component system, magnesium, biofilm formation, exopolysaccharide production, environmental stress, and Dickeya dadantii strain 3937

\section{Introduction}

Biofilm is a bacterial community encased in an extracellular matrix, which is predominantly composed of polysaccharides, in which the bacteria are imbedded (Costerton et al., 1995; Denese et al., 2000). Development of biofilm is a multistep process. In general, development pathway proceeds from the initial attachment and immobilization of bacteria to a solid surface followed by the formation of the cell cluster or micro-colony and ultimately by a threedimensional architecture (Costerton et al., 1995). Bacterial biofilms have a great significance in medical, industrial and environmental settings, for examples; bacteria in biofilms are more resistance to adverse environmental stresses such as desiccation, extreme temperature, antimicrobial agents and chlorination (Costerton et al., 1995 and 1999; Solano et al., 2002; Scher et al., 2005). Several factors have been suggested to account for biofilm resistance, for examples, slow growth, the presence of an exopolysaccharide matrix that can slow the diffusion of chemicals or inactivate antimicrobial agents, and phenotypic changes in bacteria resulting in resistance occurring within the biofilm environment (Scher et al., 2005). The biofilm forming ability of bacteria promotes host colonization and is required for virulence as shown for several animal and plant pathogenic 
bacteria (Verhoelf, 1997; Marques et al., 2002; Walker et al., 2004).

Recent molecular and genetic studies have identified several genes whose products are thought to be important for biofilm formation. For examples, biofilm formation is impaired by mutations in genes involved in flagellar or twitching-mediated motility, synthesis of exopolysaccharides, quorum sensing, outer membrane adhesins as well as global regulators of gene expression (Davies et al., 1993; Pratt and Kolter, 1998; O'Toole et al., 2000; Solano et al., 2002; Prouty and Gunn, 2003). However, no information is available about the formation of biofilm and its regulation in Dickeya dadantii (formerly Erwinia chrysanthemi) strain 3937, which is a brute-force, necrotrophic phytopathogen that macerates the plant cell wall of a wide array of dicotyledons, causing soft-rot disease by secreting multiple virulence factors (Toth et al., 2003).

Signal transduction across biological membranes is central to the ability of cells to integrate and process environmental information. In bacteria, signaling across membranes is most often accomplished by use of so called two-component systems. The PhoP-PhoQ system is a classical two-component system, where PhoP is a response regulatory protein that is necessary to transcribe more than 40 genes and PhoQ is a sensor protein that possesses both autophosphorylation and phosphatase activities in response to magnesium and calcium in animal pathogen Salmonella (Miller and Mekalanos, 1990; Garcia-Vescovi et al., 1996). It was earlier reported that the homologue of PhoP-PhoQ in $D$. dadantii strain 3937 is required for virulence, hyperinduction of pectate lyase synthesis, resistance to antimicrobial peptide, accumulation of acetyl-coenzyme A, polygalacturonase production, and motility (Haque and Tsuyumu, 2005; Haque et al., 2005; Haque et al., 2006; Haque et al., 2008). In the present article, we show that the PhoP-PhoQ two-component regulatory system is required for biofilm formation in D. dadantii strain 3937.

\section{Materials and Methods}

\subsection{Bacterial strains and culture media}

D. dadantii 3937 (wild type) and its derivative strains MH70 (km cassette inserted phoP mutant), and MH72 (km cassette inserted phoQ mutant) used in this study were described previously (Haque and Tsuyumu, 2005). The growth medium SOBG (salt-optimized broth plus glycerol) consisted of SOB plus $2 \%$ of glycerol (per liter, $20 \mathrm{~g}$ of tryptone, $5 \mathrm{~g}$ of yeast extract, $0.5 \mathrm{~g}$ of $\mathrm{NaCl}, 2.5 \mathrm{~g}$ of $\mathrm{MgSO}_{4}, 0.186 \mathrm{~g}$ of $\mathrm{KCl}$, and $50 \mathrm{ml}$ of $40 \%$ glycerol) was used to induce biofilm formation. To study the effect of magnesium on induction of biofilm formation, D. dadantii strain was grown at room temperature in SOBG medium supplemented with $0,0.1,1.0,2.5$ or $10 \mathrm{mM}$ of $\mathrm{MgSO} 4.7 \mathrm{H}_{2} 0$. The strain was also grown in yeast extractpeptone (YP) medium ( $1 \%$ of peptone, $0.5 \%$ of yeast extract, $\mathrm{pH}$ 6.8), Luria-Bertani (LB) medium ( $1 \%$ of tryptone, $0.5 \%$ of yeast extract, $0.5 \% \mathrm{NaCl}, \mathrm{pH} 7.0$ ), and $\mathrm{M} 63$ glycerol minimal medium (per liter, $2.5 \mathrm{~g}$ of $\mathrm{NaCl}, 3 \mathrm{~g}$ of $\mathrm{KH}_{2} \mathrm{PO}_{4}$, $7 \mathrm{~g}$ of $\mathrm{K}_{2} \mathrm{HPO}_{4}, 2 \mathrm{~g}$ of $\left(\mathrm{NH}_{4}\right)_{2} \mathrm{SO}_{4}, 0.5 \mathrm{mg}$ of $\mathrm{FeSO}_{4}, 1 \mathrm{~g}$ of $\mathrm{MgSO}_{4}, 2 \mathrm{~g}$ of thiamine hydrochloride, and $0.2 \%$ (wt/vol) of glycerol. When required, antibiotics were added at the following final concentrations: nalidixic acid at $50 \mu \mathrm{g} / \mathrm{ml}$, kanamycin at $50 \mu \mathrm{g} / \mathrm{ml}$, and tetracycline at $12.5 \mu \mathrm{g} / \mathrm{ml}$. The optical density of the bacterial culture was measured by Bactomonitor BACT-500 (Intertech, Tokyo, Japan) at $660 \mathrm{~nm}$.

\subsection{Biofilm formation assay}

Biofilm formation was assayed by the ability of cells to adhere to the glass test tubes, as described previously (O'Toole and Kolter, 1998a) with few modifications. Early stationary growth phase cells were diluted $(1: 10)$ in fresh SOBG medium and incubated at $27^{\circ} \mathrm{C}$ without shaking at least $72 \mathrm{~h}$. Then the cultures were 
removed from the test tubes and rinsed 2-3 times with sterile distilled water to remove loosely associated cells. A $1 \%$ of crystal violet (CV) solution (about $5.5 \mathrm{ml}$ ) was added to each glass test tube (dye stains the cells but not the glass surface) and kept at room temperature for $30 \mathrm{~min}$ then rinsed 2-3 times with sterile distilled water. At this time, the biofilm was visible as purple rings formed at the interface between the air and liquid medium. For quantitative analysis, about 2 $\mathrm{ml}$ of $95 \%$ ethanol was added to each CV-stained glass test tube then vortex vigorously to solubilize the cells. The biofilm production was measured at $600 \mathrm{~nm}$ by spectrophotometer (Spectronic, New York, USA).

\subsection{Phase contrast microscopy}

After staining of the attached bacterial cells with crystal violet $(\mathrm{CV})$, the glass test tube was examined using a phase-contrast microscope (Olympus BX51, Tokyo, Japan). Images were captured using viewfinder (Pixera, version 3.0.1).

\subsection{Quantification of exopolysaccharide}

To estimate exopolysaccharide production, strains were grown in SOBG at $27^{\circ} \mathrm{C}$ with shaking at $180 \mathrm{rpm}$ until $\mathrm{OD}_{660}$ at 1.0. One $\mathrm{ml}$ of culture was centrifuged at 40,000 rpm for $10 \mathrm{~min}$ at $4^{\circ} \mathrm{C}$. The resultant supernatant was mixed with three volume of chilled $95 \%$ ethanol and centrifuged at $100,000 \mathrm{rpm}$ for $20 \mathrm{~min}$ at $4^{\circ} \mathrm{C}$ to precipitate the exopolysaccharide. The supernatant was discarded and after drying, the pellet was resuspended in distilled water. To quantify the purified exopolysaccharide, phenolsulfuric acid method described by Hodge and Hofreiter (1962) was used.

\subsection{Real-time quantitative-PCR}

Wild type, phoP and $p h o Q$ mutants were grown in SOBG medium containing high concentrations of magnesium $(10 \mathrm{mM})$ at early stationary phase $\left(\mathrm{OD}_{660}\right.$ at 1.0$)$, then total RNA was isolated using a QIAGEN RNeasy RNA isolation kit as described by the manufacturer (QIAGEN Hilden, Germany). RNA was quantified using a NanoDroP ND-100 spectrophotometer (NanoDroP Technologies, Wilmington, DE). Real-time PCR was performed in an MX3000p Multiplex quantitative PCR system (Stratagene) using the SYBR Premix EX.Tag RT-PCR kit (TaKaRa, Japan). The transcripts of weaP, wza and $w z c$ were amplified using primers described in Table 1 . The $16 \mathrm{~S}$ rRNA gene was selected as control of experiment as it is constitutively expressed. The amount of RNA was expressed as the n-fold difference relative to the control gene $\left(2^{-\Delta \mathrm{CT}}\right.$, where $\Delta \mathrm{CT}$ represents the difference in threshold cycle between the target and control genes). The final value is represented as the mean and standard deviation of three independent experiments.

\subsection{Survival assays}

After biofilm formation (about $72 \mathrm{~h}$ after incubation) by $D$. dadantii strain 3937 , culture was removed from glass test tube, and then rinsed 2-3 times with sterile distilled water to remove loosely associated bacteria from surface of the glass test tube. To remove biofilm associated bacteria from surface of the glass test tube about $5.5 \mathrm{ml}$ of sterile distilled water was added in each test tube then vortex. Cells were then centrifuged and resuspended in sterile distilled water to a final concentration of ca $10^{7}$ colony forming unit (CFU)/ml. Logarithmic- and stationary- phase cultures of wild type was obtained by growing cells in YP to optical density of 0.6 and 1.5 , respectively under shaking condition. Cells were then centrifuged and resuspended in sterile distilled water to a final concentration of ca $10^{7} \mathrm{CFU} / \mathrm{ml}$. To test susceptibility to acidic $\mathrm{pH}$ (4.0), cells were resuspended in $1 \mathrm{ml}$ of sterile citrate-phosphate buffer, $\mathrm{pH}$ 4.0. Aliquots were taken at $1 \mathrm{~h}$, serially diluted and plated. Testing for osmotic stress resistance was similarly carried out by incubating cells in the presence of $1 \mathrm{M} \mathrm{NaCl}$. All experiments were carried out at least three times. 
Table 1. List of primers used in this study

\begin{tabular}{ccc}
\hline Name of primer & Primer sequence $\left(5^{\prime}------3\right)$ & Product length \\
\hline$w e a P$-forward & GTTGCAACGTCAGGTGAA & $275 \mathrm{bp}$ \\
$w e a$-reverse & TAATGACGAGGGAACCGA & ------- \\
$w z a$-forward & GTCAGCAACCTATCTCCA & $327 \mathrm{bp}$ \\
$w z a$-reverse & CCATCTGGTCATCCCTT & ------ \\
$w z c$-forward & GAAGCTGAGATCTCCAAG & $227 \mathrm{bp}$ \\
$w z c$-reverse & CTAGCCTTACTGATGCTC & ------ \\
16S-rRNA-forward & AGAGGATGACCAGCCACACT & Haque et al., 2005 \\
16S-rRNA-reverse & AGGTGTAGCGGTGAAATGCG & Haque et al., 2005 \\
\hline
\end{tabular}

\section{Results and Discussion}

\subsection{Biofilm formation by $D$. dadantii strain 3937 in various growth conditions}

The ability of $D$. dadantii strain 3937 to form biofilm on different abiotic surfaces such as polyvinyl chloride (PVC), polystyrene and glass test tube were examined. Polyvinyl chloride and glass test tube surface were found suitable for biofilm formation (data not shown). We investigated biofilm formation in various growth media at $27^{\circ} \mathrm{C}$ (Fig. 1). The biofilm was visualized as a purple ring of $\mathrm{CV}$-stained cells that formed at the interface between the air and the medium of a static liquid culture. $D$. dadantii strain 3937 formed biofilms in various growth media to a different extent. After $72 \mathrm{~h}$ incubation, higher and thicker biofilms were built up in SOBG medium and it could be formed biofilms in M63 glycerol minimal medium comparable to those formed on SOBG. However, a few biofilms were detected on YP and LB medium. Nutritional status of the media play an important role in biofilm formation in several other bacteria, for examples, Salmonella spp. produce thicker and denser biofilms in nutrient-limited medium, while Pseudomonas fluorescens produces better biofilms in nutrient rich medium (Song and Leff, 2006).

Next, we examined the ability of different divalent cations such as $\mathrm{Mg}^{2+}, \mathrm{Ca}^{2+}, \mathrm{Mn}^{2+}, \mathrm{Cu}^{2+}$, $\mathrm{Zn}^{2+}$ and $\mathrm{Ba}^{2+}$ and monovalent cations such as $\mathrm{Na}^{+}$and $\mathrm{K}^{+}$on biofilm formation using $1 \mathrm{mM}$ of their corresponding chloride salts. It was observed that $\mathrm{Mg}^{2+}$ greatly induced biofilm formation whereas $\mathrm{Ca}^{2+}, \mathrm{Mn}^{2+}$ and $\mathrm{Zn}^{2+}$ enhanced biofilm formation only slightly but $\mathrm{Cu}^{2+}, \mathrm{Ba}^{2+}, \mathrm{Na}^{+}$or $\mathrm{K}^{+}$failed to promote biofilm formation (data not shown). Moreover, the influence of different concentration of $\mathrm{Mg}^{2+}(0$, $0.1,1.0,2.5$ and $10 \mathrm{mM}$ ) on biofilm formation was investigated in SOBG medium at $\mathrm{pH} 7.0$ (Fig. 2). The maximal biofilm formation was observed in standard SOBG medium, where 2.5 $\mathrm{mM} \mathrm{Mg}^{2+}$ was used. The minimum concentration of $\mathrm{Mg}^{2+}$ that showed significant and positive effect for biofilm formation was $0.1 \mathrm{mM}$. However, biofilm formation was decreased when $10 \mathrm{mM} \mathrm{Mg}^{2+}$ was used than that in the medium containing $2.5 \mathrm{mM} \mathrm{Mg}{ }^{2+}$. However, the media without $\mathrm{Mg}^{2+}$, biofilm was not formed.

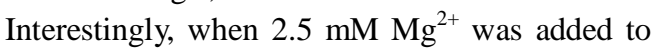
this culture, biofilm formation was recovered dramatically after $48 \mathrm{~h}$ incubation period (data not shown). It is worthy to note that biofilm formation was thoroughly inhibited when grown in the presence of $2.5 \mathrm{mM} \mathrm{Mg}{ }^{2+}$ together with 0.5 M EDTA or more (data not shown). This result suggests that biofilm inhibition of $D$. dadantii 3937 by EDTA is due to chelating of $\mathrm{Mg}^{2+}$. The growth rate of $D$. dadantii strain 3937 was indistinguishable with respect to the concentrations ( 0 to $10 \mathrm{mM}$ ) of $\mathrm{Mg}^{2+}$ (data not shown). Thus, lower level of biofilm formation by $D$. dadantii strain 3937 in YP or in LB medium may be, at least in part, due to lack of magnesium in these media. 


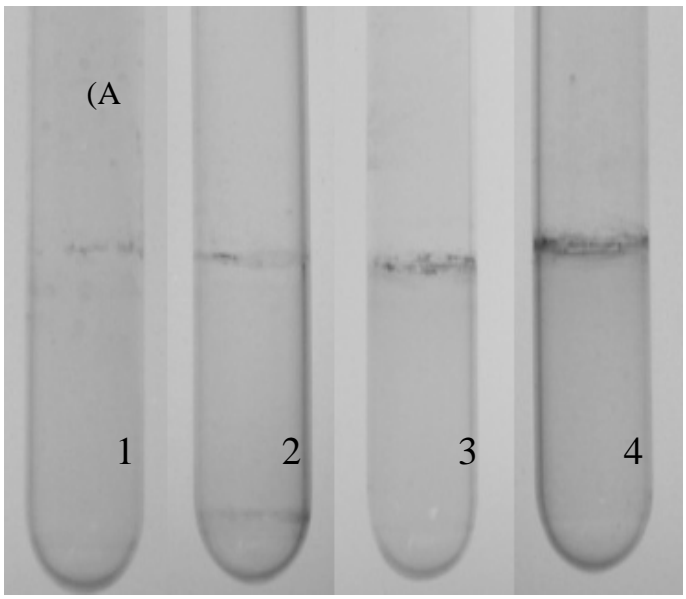

(B)

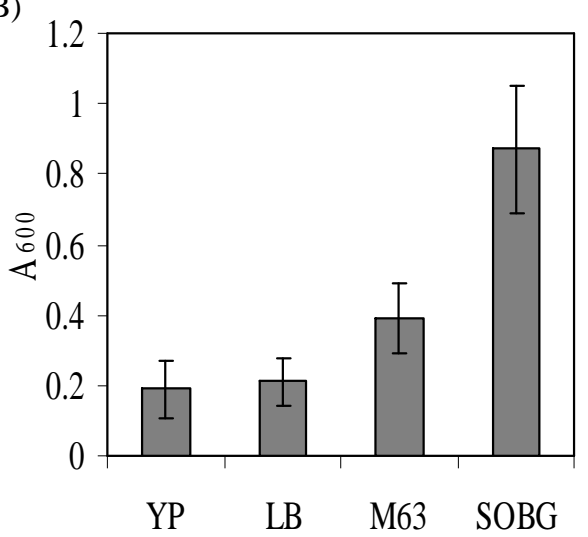

Fig. 1. Effect of different media on biofilm formation by $D$. dadantii strain 3937 in glass test tube $(1=\mathrm{YP}, 2=\mathrm{LB}, 3$ $=$ M63 glycerol minimal medium and $4=$ SOBG). The extent of biofilm formation after $72 \mathrm{~h}$ of incubation in the indicated media (A) and quantification of biofilms as the absorbance at $600 \mathrm{~nm}$ (B). Data are expressed as the mean of five independent experiments. Error bars indicate the standard deviation $( \pm \mathrm{SD})$.

(A

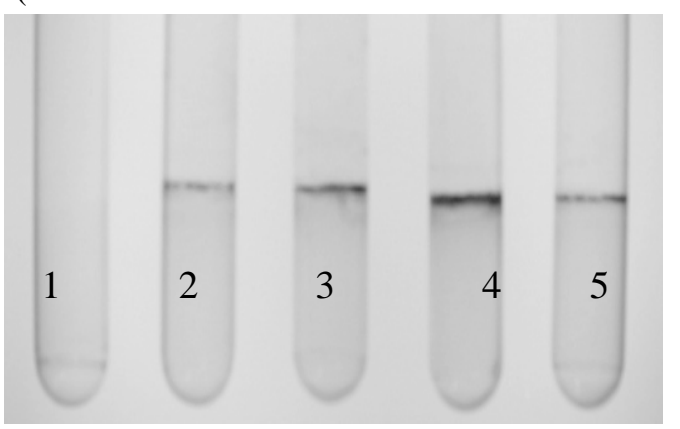

(B)

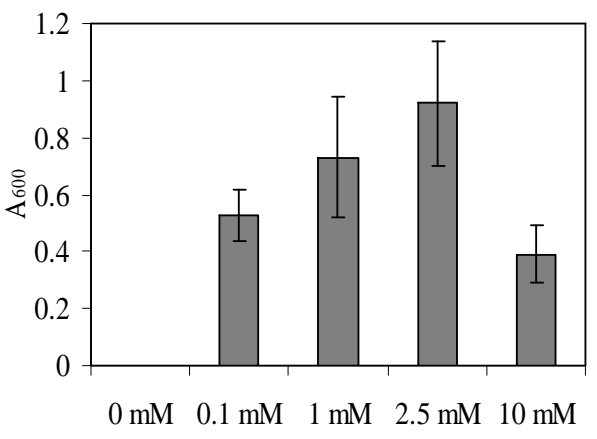

Fig. 2. Effect of different concentrations of magnesium $\left(\mathrm{MgSO}_{4} \cdot 7 \mathrm{H}_{2} \mathrm{O}\right)$ on biofilm formation of $D$. dadantii strain 3937 in glass test tube $(1=0 \mathrm{mM}, 2=0.1 \mathrm{mM}, 3=1.0 \mathrm{mM}, 4=2.5 \mathrm{mM}$ and $5=10 \mathrm{mM})$. Biofilms were stained with crystal violet $72 \mathrm{~h}$ after incubation (A). To quantify, biofilms are solubilized in $95 \%$ ethanol then optical density of the resultant solution was measured at 600 (B). Data are expressed as the mean of five independent experiments. Error bars indicate the standard deviation $( \pm \mathrm{SD})$.

It has been demonstrated that divalent cations including magnesium and calcium influence biofilm formation directly through their effect on electrostatic interactions and indirectly via physiology-dependent attachment processes by acting as important cellular cations and enzyme cofactors (Fletcher, 1998). Moreover, electrostatic interactions contribute to biofilm cohesion, and cations are significant cross linkers of the biofilm matrix because they contribute to the integrity and stability of the outer membranes of the bacteria and properties of the lipopolysaccharides (Geesey et al., 2000). Furthermore, Costerton and associates (1995) have been reported that high concentration of magnesium might contribute to an increase in 
exopolysaccharide production and biofilm stabilization. They also stated that magnesium increased the abundance of attached cells, possibly by reducing the repulsive force between the negatively charged bacterial and substratum surfaces and between negative groups on the polysaccharides, which constitute the structure of biofilms.

\subsection{The PhoP-PhoQ two-component regulatory system repressed biofilm formation}

Previously, we have shown that multiple virulence-associated traits are repressed by the PhoP-PhoQ two-component system of $D$. dadantii strain 3937 in response to high concentration of magnesium (Haque and Tsuyumu, 2005; Haque et al., 2005; Haque et al., 2006; Haque et al., 2008). In this study, both the mutants lacking functional $p h o P$ and $p h o Q$ were formed thicker and denser biofilms in SOBG medium containing high concentration of magnesium $(10 \mathrm{mM})$ compare to that by wildtype strain in the surface of glass test tube (Fig. $3 \mathrm{~A})$. When biofilm formed on the surface of the glass test tube was examined under phasecontrast microscopy, both the mutants of phoP and $p h o Q$ formed large clusters of the cells closely adhered with each other covering almost the entire surface of the glass. In contrast, wildtype strain formed small clusters of cells as a scattered pattern leaving wide regions empty (Fig. 3B). However, no obvious difference was observed in the number of attached cells between the phoP and phoQ mutants. When formation of biofilm was quantified, wild-type strain showed about the one third of its absorbance as compare to that by the mutants of $p h o P$ and $p h o Q$ (Fig. 3C). Thus, PhoP-PhoQ two-component regulatory system seems to negatively regulate biofilm formation in $D$. dadantii strain 3937 in response to high concentration of magnesium. In Salmonella, high concentration of magnesium stimulated the PhoQ phosphatase activity that dephosphorylates phospho-PhoP. This dephophorylation then represses the transcription of PhoP-regulated genes and derepresses PhoPrepressed genes (Castelli et al., 2000). In this study, biofilm formation was repressed by the PhoP-PhoQ two-component system in response to high concentration of magnesium, suggesting that high concentration of magnesium might interact with the periplasmic domain of PhoQ shifting the balance toward a phosphatasedominant state of the sensor protein. Under this condition PhoP would become dephosphorylated, and the expression of the PhoP-activated genes would be shut down. This/these gene(s) may lead to $D$. dadantii strain 3937 to repressed biofilm formation.

\subsection{PhoP-PhoQ two-component system upregulates exopolysaccharide production and genes responsible for exopolysaccharide biosynthesis}

It has been reported that exopolysaccharide play an important role in biofilm formation in different animal pathogenic enterobacteria such as Salmonella typhimurium and Escherichia coli (Ross et al., 1991; Solano et al., 2002; Danese et al., 2000). To examine, if $p h o P$ and $p h o Q$ has any effect on exopolysaccharide production, the mutants of $p h o P$ and $p h o Q$ as well as wild-type strain were grown in SOBG medium containing high concentration of magnesium until early stationary phase $\left(\mathrm{OD}_{660}\right.$ at 1.0$)$ then exopolysaccharide production was determined. Production of expolysaccharide by the mutants of $p h o P$ and $p h o Q$ was $0.93 \pm 0.15 \mathrm{mg} / \mathrm{ml}$ and $1.12 \pm 0.22 \mathrm{mg} / \mathrm{ml}$, respectively whereas exopolysaccharide production by the wild-type strain was $0.61 \pm 0.12 \mathrm{mg} / \mathrm{ml}$. In order to identify gene(s) responsible for exopolysaccharide production that may be regulated by $p h o P$ and $p h o Q$, the transcriptional profile of $D$. dadantii 3937 mutant strains lacking functional phoP and $p h o Q$ were compared with that of the wild-type strain in SOBG medium containing high concentration of magnesium by real time quantitative polymerase chain reaction (Fig. 4). The transcript levels of weaP, wza and wzc were upregulated in the phoP background than that of the wild type. Similar upregulation was also observed in $p h o Q$ mutant background (data not shown). 

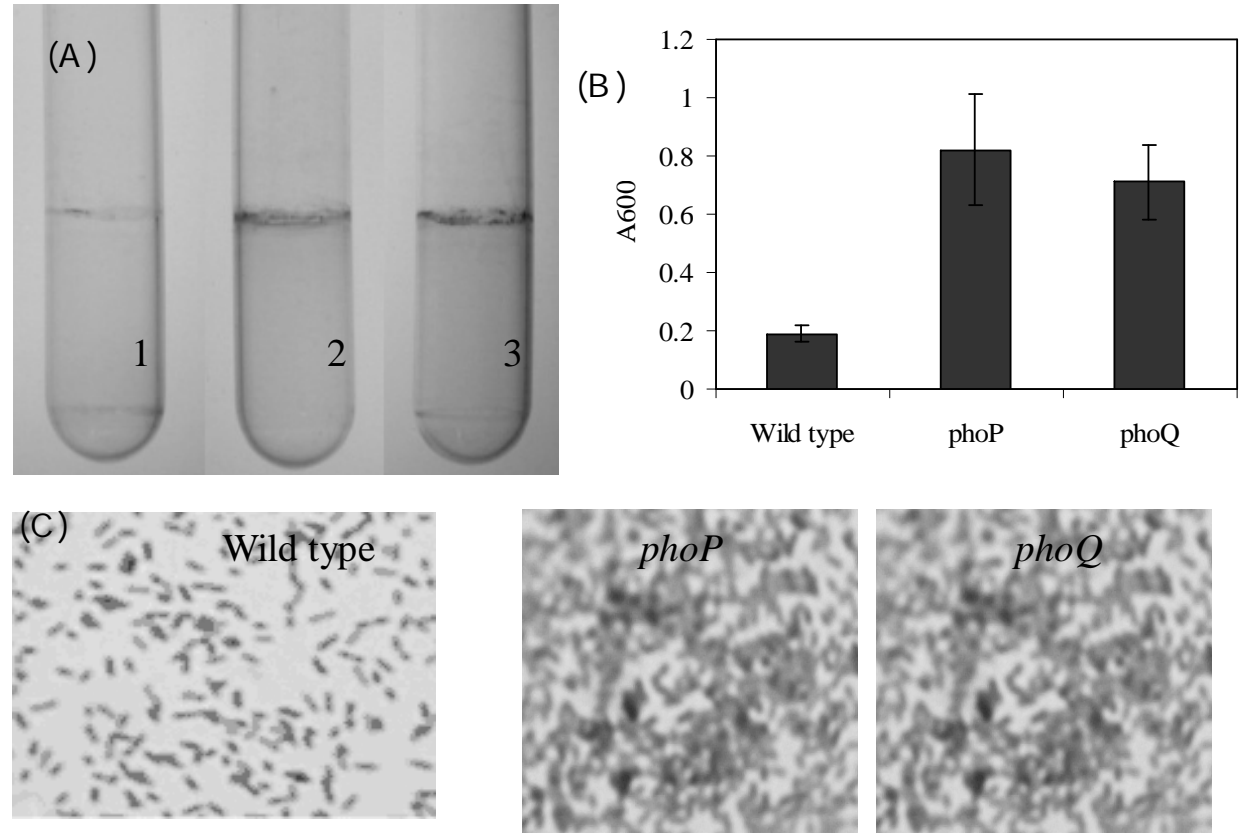

Fig. 3. Biofilms formed by different strains $(1=$ wild type, $2=p h o P$ mutant and $3=p h o Q$ mutant $)$ in glass test tube (A), quantification (B) and visual observation of biofilms by phase contrast microscopy after $72 \mathrm{~h}$ incubation (C).

Motility has been shown to be involved in biofilm formation (O'Toole and Kolter, 1998b; Pratt and Kolter, 1998; Choy et al., 2004). In case of $P$. fluorescens, the flagellum itself was reported to play a direct role in adhesion (Lawrence et al., 1987). However, Hossain and Tsuyumu (2006) observed that not only the aflagellate mutant $(\triangle f l i C)$ but also the paralyzed mutant $(\Delta m o t A)$ were equally reduced in their ability to form a biofilm, suggesting that functional flagella rather than nonfunctional flagella, are involved in biofilm formation. Moreover, mutation of gene involved in exopolysaccharide production has been shown to inability to form a biofilm (Danese et al., 2000; O'Toole et al., 2000). Recently, we reported that genes responsible for flagellum biosynthesis (fliD, fliC and fliA) are transcriptionally controlled by the PhoP-PhoQ two-component system in responding to magnesium (Haque et al., 2008). In this study, exopolysaccharide production and its genes (weaP, wza and wzc) are controlled by the PhoP-PhoQ two-component system (Fig. 4). These results, together with our previous study, suggest that the PhoP-PhoQ twocomponent system may be regulated formation of biofilm by transcriptional regulation of genes responsible for exopolysaccharide production in addition to genes responsible for bacterial motility of $D$. dadantii strain 3937 .

\subsection{Role of biofilm cells in virulence and survival in unfavorable environment}

It has been reported that biofilm cells are resistant to various environmental stresses (Scher et al., 2005; Solano et al., 2002; Elkins et al., 1999). To determine whether biofilms cells of $D$. dadantii strain 3937 showed increased resistance to logarithmic- and stationary-phase growing cells, survival assays were carried out in acidic $\mathrm{pH}$ (4.0) and high osmotic stress because when D. dadantii strain 3937 infect a plant, it confronts an acidic $\mathrm{pH}$ in the intercellular apoplastic fluid. 


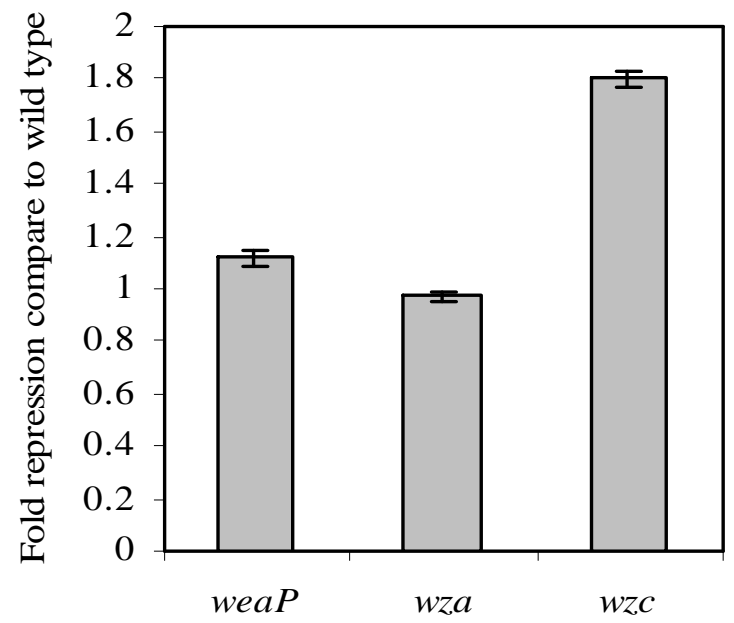

Fig. 4. Analyses of genes responsible for exopolysaccharide production (EPS) by real time quantitative polymerase chain reaction. Expressed as the ratio of the specific gene, expression level in the phoP mutant background compared to that in the wild type normalized to the level of expression of the $16 \mathrm{~S}$ rRNA gene.

(A)

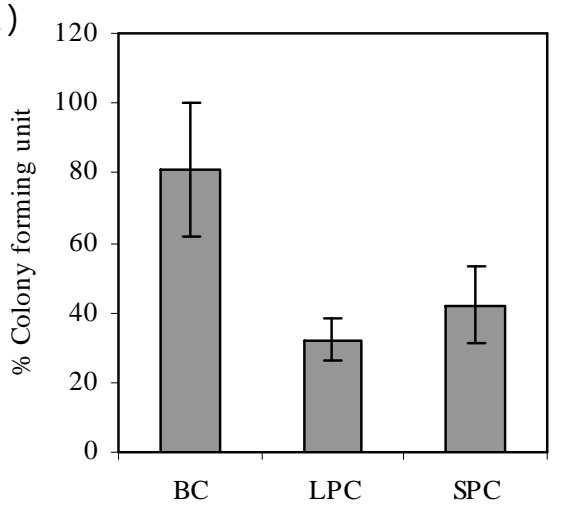

(B)

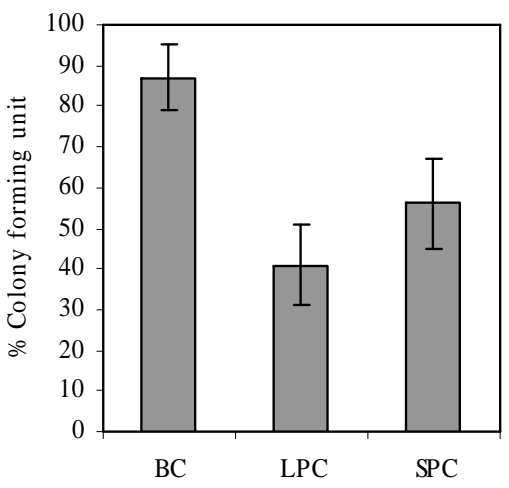

Fig. 5. Effect of acidic $\mathrm{pH}, 4.0$ (A) and high osmolarity (1 M NaCl) on susceptibility to biofilm cells (BC), logarithmic phase cells (LPC) and stationary phase cells (SPC) of D. dadantii strain 3937. For susceptibility tests, the number of colony forming units (CFU) were compared with standard YP and setting at $100 \%$. All experiments were carried out at least three times.

However, disruption of cells after maceration probably provokes an increase in the osmolarity of the infected plant tissue. It was observed that cells in the biofilm are more tolerant to acidic $\mathrm{pH}$ and high osmotic stress as compare to logarithmic- and stationary-phase cells (Fig. 5).
When virulence of biofilm cells compared with logarithmic- and stationary-phase growing cells, no difference was observed (data not shown). Thus, formation of biofilm may be an important factor for the survival of $D$. dadantii strain 3937 in natural environment. 


\section{Conclusions}

PhoP-PhoQ two-component system repressed biofilm formation in the phytopathogen $D$. dadantii strain 3937 in response to high concentration of magnesium. Accordingly, exopolysaccharide production and genes responsible for exopolysaccharide production are markedly upregulated in both $p h o P$ and $p h o Q$ mutant backgrounds than the parental strain. These results, together with our previous study, suggest that the PhoP-PhoQ two-component system may regulate formation of biofilm by transcriptional control of genes responsible for exopolysaccharide production in addition to genes responsible for bacterial motility of $D$. dadantii strain 3937 in responding to magnesium. In addition, biofilm cells showed more tolerant to acidic $\mathrm{pH}$ and high osmolarity, thus, formation of biofilm at least may protect $D$. dadantii strain 3937 from various adverse environmental cues.

\section{References}

Castelli, M. E., Garcia-Vescovi, E. and Soncini, F. C. 2000. The phosphatase activity is the target for $\mathrm{Mg}^{2+}$ regulation of the sensor protein PhoQ in Salmonella. Journal of Biological Chemistry, 30: 22948-22954.

Choy, W. K., Zhou, L., Syn, C. K., Zhang, L. H. and Swarup, S. 2004. MorA defines a new class of regulators affecting flagellar development and biofilm formation in adverse Pseudomonas species. Journal of Bacteriology, 186: 7221-7228.

Costerton, J. W., Lewandowski, Z., Caldwell, D. E., Korber, D. R. and Lappin-Scott, H. M. 1995. Microbial biofilms. Annual Review of Microbiology, 49: 711-745.

Costerton, J. W., Stewart, P. S. and Greenberg, E. P. 1999. Bacterial biofilms: a common cause of persistent infections. Science, 284: 1318-1322.

Danese, P. N., Pratt, L. A. and Kolter, R. 2000. Exopolysaccharide production is required for development of Echerichia coli K-12 biofilm architecture. Journal of Bacteriology, 182: 3593-3596.
Davies, D. G., Chakrabarty, A. M. and Geesey, G. G. 1993. Exopolysaccharide production in biofilms: substratum activation of alginate gene expression by Pseudomonas aeruginosa. Applied and Environmental Microbiology, 59: 1181-1186.

Elkins, J. G., Hassett, D. J., Stewart, P. S., Schweizer, H. P. and McDermott, T. R. 1999. Protective role of catalase in Pseudomonas aeruginosa biofilm resistance to hydrogen peroxide. Applied and Environmental Microbiology, 65: 4594-4600.

Fletcher, M. 1988. Attachment of Pseudomonas fluorescens to glass and influence of electrolytes on bacterium-substratum separation distance. Journal of Bacteriology, 170: 2027-2030.

Garcia-Vescovi, E., Soncini, F. C. and Groisman, E. A. $1996 . \mathrm{Mg}^{2+}$ as an extracellular signal: environmental regulation of Salmonella virulence. Cell, 84: 165-174.

Geesey, G. G., Wigglesworth-Cooksey, B. and Cooksey, K. E. 2000. Influence of calcium and other cations on surface adhesion of bacteria and diatoms: a review. Biofouling, 15: 195-205.

Haque, M. M., Yamazaki, A. and Tsuyumu, S. 2005. Virulence, accumulation of acetylcoenzyme A, and pectate lyase synthesis are controlled by PhoP-PhoQ twocomponent regulatory system responding to organic acids of Erwinia chrysanthemi 3937. Journal of General Plant Pathology, 71: 133-138.

Haque, M. M. and Tsuyumu, S. 2005. Virulence, resistance to magainin II and expression of pectate lyase are controlled by the PhoPPhoQ two-component regulatory system responding to $\mathrm{pH}$ and magnesium in Erwinia chrysanthemi 3937. Journal of General Plant Pathology, 71: 47-53.

Haque, M. M., Gomes, I. and Tsuyumu, S. 2006. Functional analysis of the PhoP-PhoQ twocomponent system in Erwinia chrysanthemi 
3937. Bangladesh Journal of Microbiology, 23: $38-42$.

Haque, M. M., Nahar, K., Rahim, M. A., Gomes, I. and Tsuyumu, S. 2008. PhoP-PhoQ twocomponent system required for colonization leading to virulence of Dickeya dadantii 3937 in planta. Bangladesh Journal of Microbiology, 25: 36-40.

Hodge, J. E. and Hofreiter, B. T. 1962. Determination of reducing sugars and carbohydrates. In: Whistler, R. L., and M. L. Wolfrom (ED). Methods in Carbohydrate Chemistry. Academic Press, New York. p. 388.

Hossain, M. M. and Tsuyumu, S. 2006. Flagellamediated motility is required for biofilm formation by Erwinia carotovora subsp. carotovora. Journal of General Plant Patholology, 72: 34-39.

Lawrence, J. R, Delaquis, P. J., Korber, D. R. and Caldwell, S. E. 1987. Behavior of Pseudomonas fluorescens within the hydrodynamic boundary layers of surface microenvironments. Microbial Ecology, 14: 1-14.

Marques, L. L. R., Ceri, H., Manfio, G P., Reid, D. M. and Olson, M. E. 2002. Characterization of biofilm formation by Xylella fastidiosa in vitro. Plant Disease, 86: 633-638.

Miller, S. I. and Mekalanos, J. J. 1990. Constitutive expression of the phoP regulon attenuates Salmonella virulence and survival within macrophages. Journal of Bacteriology, 172: 2485-2490.

O'Toole, G A. and Kolter, R. 1998a. Initiation of biofilm formation in Psudomonas fluorescens WCS365 proceeds via multiple, convergent signaling pathways: a genetic analysis. Molecular Microbiology, 28: 449-461.

O’Toole, G. A. and Kolter, R. 1998b. Flagellar and twitching motility are necessary for Pseudomonas aeruginosa biofilm development. Molecular Microbiology, 30: 295-304.

O’Toole, G. A., Gibbs, K. A., Hager, P. W., Phibbs, P. V. Jr. and Kolter, R. 2000. The global carbon metabolism regulator $\mathrm{Crc}$ is a component of a signal transduction pathway required for biofilm development by Pseudomonas aeruginosa. Journal of Bacteriology, 182: 425-431.

Pratt, L. A. and Kolter, R. 1998. Genetic analysis of Escherichia coli biofilm formation: roles of flagella, motility, chemotaxis and type I pili. Molecular Microbiology. 30: 285-293.

Prouty, A. M. and Gunn, J. S. 2003. Comparative analysis of Salmonella enterica Serovar Typhimurium biofilm formation on gallstones and on glass. Infection and Immunity, 71: 7154-7158.

Ross, P., Mayer, R. and Benziman, M. 1991. Cellulose biosynthesis and function in bacteria. Microbiology Review, 55: 279-281.

Scher, K, Römling, U. and Yaron, S. 2005. Effect of heat, acidification, and chlorination on Salmonella enterica serovar Typhimurium cells in a biofilm formed at the air-liquid interface. Applied and Environmental Microbiology, 71: 1163-1168.

Solano, C., Garcia, B., Valle, J., Berasain, C., Ghigo, J. M., Gamazo, C. and Lasa, I. 2002. Genetic analysis of Salmonella enteritidis biofilm formation: critical role of cellulose. Molecular Microbiology, 43: 793-808.

Song, B. and Leff, L. G. 2006. Influence of magnesium ions on biofilm formation by Pseudomonas fluorescens. Microbiological Research, 161: 355-361.

Toth, I. K., Bell, K., Holeva, M. C. and Birch, P. R. J. 2003. Soft rot erwiniae: from genes to genomes. Molecular Plant Pathology, 4: 17-30.

Verhoef, J. 1997. Host defense against infection. In: Crossley K. B., G. L. Archer (ED). The Staphylococci in Human Disease. Churchill Livingstone, NY pp 213-232.

Walker, T. S., Bais, H. P., Deziel, E., Schweizer, H. P., Rahme, L. G, Fall, R. and Vivanco, J. M. 2004. Pseudomonas aeruginosa-plant root interactions: Pathogenicity, biofilm formation, and root exudation. Plant Physiology, 134: 320-331. 
